\title{
Ubiquitous Mechanisms of Energy Dissipation in Noncontact Atomic Force Microscopy
}

\author{
S. Alireza Ghasemi, ${ }^{1}$ Stefan Goedecker, ${ }^{1}$ Alexis Baratoff, ${ }^{1}$ Thomas Lenosky, ${ }^{2}$ Ernst Meyer, ${ }^{1}$ and Hans J. Hug ${ }^{1,3}$ \\ ${ }^{1}$ Department of Physics and National Center for Research in Nanoscale Science, University of Basel, \\ Klingelbergstrasse 82, 4056 Basel, Switzerland \\ ${ }^{2}$ Department of Materials Science and Engineering, Ohio State University, Columbus, Ohio 43210, USA \\ ${ }^{3}$ Swiss Federal Laboratory for Materials Testing and Research, 8600 Dubendorf, Switzerland
} (Received 18 February 2008; published 13 June 2008)

\begin{abstract}
Atomistic simulations considering larger tip structures than hitherto assumed reveal novel dissipation mechanisms in noncontact atomic force microscopy. The potential energy surfaces of realistic silicon tips exhibit many energetically close local minima that correspond to different structures. Most of them easily deform, thus causing dissipation arising from hysteresis in force versus distance characteristics. Furthermore, saddle points which connect local minima can suddenly switch to connect different minima. Configurations driven into metastability by the tip motion can thus suddenly access lower energy structures when thermal activation becomes allowed within the time required to detect the resulting average dissipation.
\end{abstract}

Noncontact (actually near-contact) atomic force microscopy (NCAFM) [1] is becoming an established technique in nanoscience which, unlike scanning tunneling microscopy, can resolve atomic-scale features even on insulating samples. Recent work has proven that controlled manipulation of individual atoms is also possible using NCAFM [2]. These capabilities will, e.g., enable quantitative studies of externally triggered conformational changes of individual molecules assembled on various substrates. Existing models cannot, however, explain the following facts. First, energy dissipation has been observed in many NCAFM experiments with atomic resolution on different samples. Second, dissipation images often exhibit sudden contrast changes while simultaneously recorded topography images are much less affected. Thus dissipation in NCAFM sensitively depends on the "tip state" in a hitherto poorly understood fashion [3]. Our work explains these ubiquitous features and thus contributes to the general understanding of tip-induced dissipation processes in atomic-scale scanning probe investigations.

In most NCAFM experiments, a microfabricated high- $Q$ silicon cantilever is driven at its fundamental flexural resonance. Atomic resolution of surface features is achieved if the sharp tip at the cantilever end comes within the range of short-range chemical and/or steric interactions with the sample. The total tip-sample interaction causes a frequency shift $\Delta f$ which can be kept constant in order to record a topographic image. The average energy $\Delta E$ dissipated per cycle arising from nonadiabatic relaxation processes induced by time-dependent tip-sample forces can be simultaneously measured [4]. The net work done per cycle by the cantilever oscillating perpendicular to the surface is given by

$$
W=-\oint F(z) d z
$$

where the integral is taken over one oscillation, $z$ is the vertical distance between the outermost atoms of the tip and the surface, ignoring relaxation effects, while $F(z)$ is the $z$ component of the force acting on the tip, including those effects. This is reasonable because $z$ tracks the motion of the cantilever end, while changes in $F(z)$ are typically transmitted within $\sim 1$ ns through the tip. Possible dissipation mechanisms have been reviewed [3]; typical values of $\Delta E \sim 0.1-1 \mathrm{eV}$ per cycle have been observed under stable imaging conditions. They can be attributed to one or a few sample atoms rapidly jumping back and forth upon tip approach and retraction below a site-dependent critical distance $z_{c}$. At zero temperature, a jump $\Delta V_{i}<0$ occurs in the potential energy $V(z)$ of the tip-sample system whenever the system rearranges. The current potential minimum then disappears together with the energy barrier (a saddle point in configuration space) separating it from another lower minimum. Because $F(z)=-\partial V / \partial z$ between successive jumps, Eq. (1) implies that $W=-\sum_{i} \Delta V_{i}$. The energy released at each jump $i$ excites vibrations which eventually decay; i.e., energy is dissipated. Energy conservation implies $\Delta E=W$ on average. Typical vibrational relaxation times being less than $1 \mathrm{~ns}$, jumps can be considered as instantaneous. They lead to a hysteretic force $F(z)$ in each cycle and, at zero bath temperature, to a step in $\Delta f$ and to a stepwise increase of $\Delta E$ at $z_{c}$ [5]. At finite temperature, such jumps can be thermally activated before the relevant barrier disappears, i.e., slightly above $z_{c}$. The hysteresis is thereby reduced, and the steps in $\Delta f$ and $\Delta E$ are smeared on average [6], The finite response times of the measurement electronics lead to similar effects [7].

Recent atomistic simulations have focused on jumps of sample atoms induced by the tip on (001) cleavage planes of ionic crystals [7-9] or on reconstructed $\mathrm{Si}$ and $\mathrm{Ge}$ surfaces $[2,10-12]$. However, these simulations assumed rather small $\mathrm{MgO}$ or $\mathrm{Si}$ clusters as model tips. Energy dissipation then arises from instabilities in the positions 
of sample atoms subjected to short-range interactions. Such scenarios require a particular combination of tip and sample properties and therefore cannot explain dissipation in general. In the following we show that relaxation and rearrangements within more realistic silicon tips can also lead to hysteresis, hence additional dissipation. This mechanism is active on almost any sample. In order to study these effects, we have generated several thousand structures of model $\mathrm{Si}_{72} \mathrm{H}_{50}$ tips using the minima hopping algorithm [13] and selected more than 30 low-energy configurations for a more detailed study. Then we computed their total energies $V(z)$ in a few quasistatic approach-retraction cycles of the tip down to $z=3.1 \AA$ above the up atom of a tilted dimer on the prerelaxed $p(2 \times$ 1) reconstructed (001) surface of a slab with all Si atoms fixed. Twenty-five silicon atoms near the tip apex were allowed to relax, whereas atoms in the base of the tip were kept fixed. This allows comparison with simulations of the same system, which assumed a small $\mathrm{Si}_{10} \mathrm{H}_{15}$ tip but allowed relaxation of surface layers $[10,11]$. Our computations were performed with a tight-binding model, composed of the well-known Lenosky et al. tight-binding parameters for Si [14], together with additional parameters fitted using a similar methodology to accurately model Si$\mathrm{H}$ and $\mathrm{H}-\mathrm{H}$ interactions relevant for hydrogen terminated surfaces and clusters.

In practice a sharp reactive silicon tip obtained, e.g., by sputtering off the native oxide, exposes many atoms that cannot be in the preferred fourfold coordination environment. A large number of distorted structures have very similar energies. Six tip structures with energies within an interval of $0.1 \mathrm{eV}$ are displayed in Fig. 1. In the case of free $\mathrm{Si}_{N}$ clusters $(N \geq 13)$, similar small energy differences have also been found in density functional or even more accurate quantum Monte Carlo calculations [15]. This is thus certainly not an artifact of the tight-binding scheme, but a general property of such frustrated covalently bonded systems.

Our model tips have a fixed crystalline base which enforces a pyramidal shape. However, several threefold coordinated atoms with dangling bonds, themselves bonded to threefold or fourfold coordinated atoms, with strained bonds and bond angles invariably occur around the apex. Tips with one protruding atom facing an up dimer atom produce $V(z)$ curves with one downward jump upon approach and retraction, respectively, without transfer of atoms in the investigated range. However, as shown in Fig. 2, the initial cycle is sometimes different from subsequent ones. This implies a dynamical stabilization of certain tip configurations. In most runs, this stabilization is established from the start. An example is shown in Fig. 3. Corresponding values of $\Delta E$ ranged between 0 and about $0.5 \mathrm{eV}$ per cycle. By contrast, a unique $F(z)$ was found with a small adatom-terminated Si tip above an up dimer atom on the $c(4 \times 2) \mathrm{Si}(001)$ surface, whereas a hysteretic $F(z)$ leading to $\Delta E=0.3 \mathrm{eV}$ was found above a down dimer atom $[10,11]$. In those studies the main contribution to $\Delta E$ was attributed to dimer flipping, while it is solely due to tip deformation in our case. A semiquantitative comparison between both studies appears justified. Indeed, both the dimer flip in Refs. $[10,11]$ and the tip deformation in our case are triggered by bond formation between the outermost tip atom and the nearest surface atom. The maximum attractive force was $-2.75 \mathrm{nN}$ at $z=3.2 \AA$ in the former computation [10], compared to $-3.4 \pm 0.2 \mathrm{nN}$ at $3.3 \pm$ $0.1 \AA$ achieved on the nearly linear $V(z)$ branch which appears at short distances in our stabilized cycles.

By analogy with the behavior of a soft cantilever subject to adhesive forces, the observed jumps (which occur whenever the force gradient exceeds the stiffness of the tip) are larger if the tip is softer. The large spread in tip stiffness, hence in $\Delta E$, arises because of the above-mentioned differences in the coordination of backbonded atoms and of the resulting strains in the amorphouslike apex structure. We surmise that if surface dimer relaxation were allowed, $\Delta E$ would still be dominated by tip deformation, at least for the softest tip structures.

Let us next discuss effects due to a finite temperature $T$. As already pointed out and discussed in detail for sample atoms displaced by the tip [2,6-12], thermal activation
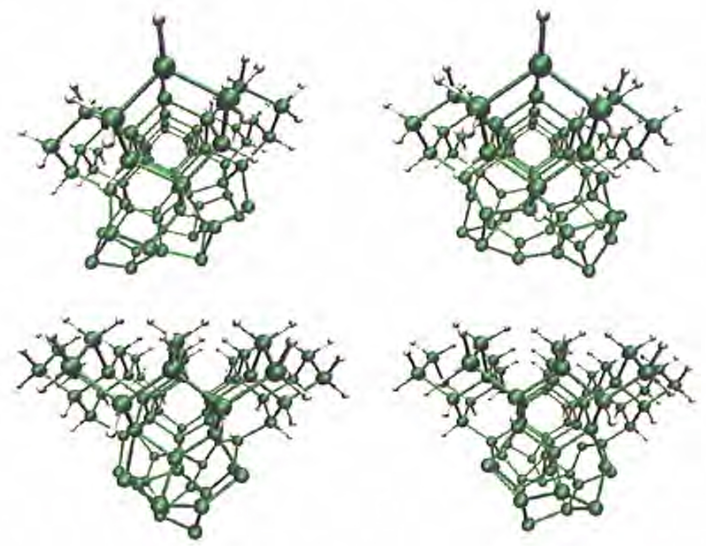

FIG. 1 (color online). Representative low-energy tip structures used in our simulations [dark gray (green): Si atoms; light gray: $\mathrm{H}$ atoms passivating bulklike Si layers). 


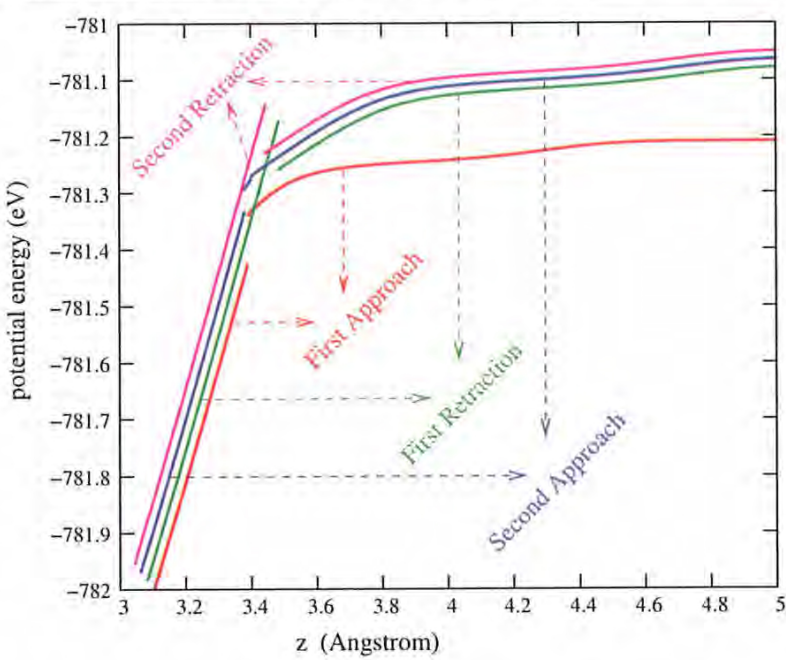

FIG. 2 (color online), Potential energy versus unrelaxed tipsample distance $z$ for two subsequent approaches and retractions. The first retraction and the second cycle curves are slightly shifted to the left (by steps of $0.02 \AA$ ) and upwards (by steps of $0.015 \mathrm{eV}$ ) to clarify the figure.

over energy barriers which vanish at the end points of $V(z)$ branches like those in Figs. 2 and 3 allow highly nonequilibrium populations of those branches to relax into populations that are closer to thermal equilibrium. The importance of such effects can be assessed by comparing the mean residence times in local energy minima with time scales relevant in NCAFM experiments $[3,7,9,16]$. Transition state theory [17] predicts a mean jump rate out of a local energy minimum of

$$
\nu=\nu_{0} \exp \left[-E_{a} /\left(k_{B} T\right)\right]
$$

where $E_{a}$ is the energy barrier to be surmounted and the attempt frequency $\nu_{0}$ is roughly $10^{13} \mathrm{~Hz}$. The following estimates refer to room temperature, but can be scaled if desired using Eq. (2). One relevant scale is determined by the time during which the tip can strongly interact with a surface atom. Our simulations, as well as previous ones, show that this occurs within a range $d \sim 1 \AA$ around the turning point of the tip. For a typical oscillation amplitude $A \sim 100 \AA$ at a frequency $f \sim 100 \mathrm{kHz}$, the "interaction time" is $\sqrt{(d / A)} / f \sim 10^{-6} \mathrm{~s}$. Within this time barriers of up to $0.41 \mathrm{eV}$ can be surmounted. The corresponding threshold would be $0.45 \mathrm{eV}$ if a whole oscillation period were considered. Another relevant scale is the response time of the amplitude controller over which dissipation is effectively sampled (at least $\sim 10^{-3} \mathrm{~s}$ ). Within this interval a $0.58 \mathrm{eV}$ barrier could be surmounted, leading to a hysteresis loop in one out of 100 cycles and a 100 times lower average dissipation. To reduce noise, measurements are made at closely spaced positions over an averaging time $t_{\mathrm{avg}}$ of up to $0.1 \mathrm{~s}$; dissipation arising from jumps over

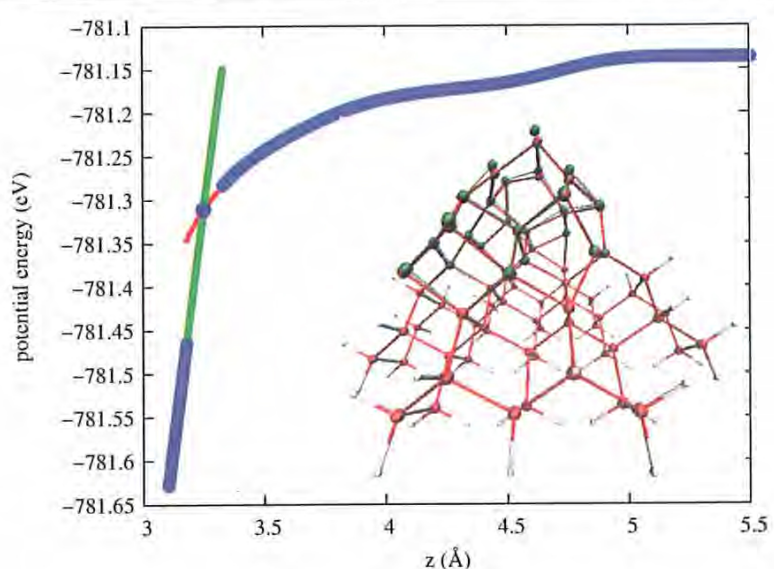

FIG. 3 (color online). Representative $V(z)$ variation for a stable set of tip structures. Inset: Structures corresponding to the two configurations near the crossing [thin (red) line: approaches; medium (green) line: retractions; thick (blue) line: coinciding segments].

barriers less than $0.70 \mathrm{eV}$ could then be measured. On the other hand, individual jumps over higher barriers would be detectable in real time, either as telegraphlike noise [18] or as sudden changes in image contrast [2,7,9]. Hereafter, attention is focused on situations where only continuous dissipation is measured.

Barriers to flip a single dimer were estimated to be 0.12 and $0.20 \mathrm{eV}$ for the $p(2 \times 1)$ and $c(4 \times 2)$ reconstructions of the $\mathrm{Si}(001)$ surface, respectively [11], and similar barrier heights are expected for the dimerlike configurations at the surfaces of our tip structures. Deeper inside the tip, barriers existing for amorphous silicon may be more appropriate. Starting from well-relaxed configurations of that material, an extensive search yielded a $2.4 \mathrm{eV}$ wide distribution of the lowest barriers with a maximum around $3 \mathrm{eV}$ extending down to zero [19]. Judging from Fig. 2 and estimates in that work, roughly one energy barrier below $0.5 \mathrm{eV}$ per relaxed atom is expected.

Owing to interaction with the surface, the energies of all tip structures vary as a function of $z$ (see Fig. 2), hence time. Moreover, energy differences between different tip configurations also change and the corresponding $V(z)$ curves can even cross. The lowest energy barriers connecting those configurations also vary. Using an "improved dimer method" [20], we therefore initiated searches for the lowest barriers connecting a few of our low-energy tip structures to other structures as a function of decreasing $z$. In the process we found many barriers, several between 0.4 and $0.7 \mathrm{eV}$, for distances somewhat larger than $z_{c}$. Thus activated transitions between different tip structures can occur at room temperature even before the $V(z)$ branch corresponding to bond formation is reached. Moreover, during the tip motion, the same saddle point can suddenly connect one of the two connected minima to a different one 
or even connect a new pair of local minima. Such a switching of the connectivity can occur if the energy landscape of a complex system [21] is qualitatively changed by an applied bias, e.g., the tip-sample interaction in our case. Changes in connectivity thus happen at distances where the tip-sample interaction is weaker than that needed for a minimum to disappear.

If all barriers were significantly smaller than $0.4 \mathrm{eV}$, configurations corresponding to different local minima would be maintained in thermal equilibrium by frequent jumps during each cycle, and negligible dissipation would occur. By analogy with reacting chemical species (see, for instance, [22]), it would then be sufficient to replace $V(z)$ by the free energy, i.e., a sum over stable tip structures weighted by Boltzmann population factors times local vibrational free energies. If weights $<0.01$ are considered negligible, only structures with energies differing by less than $0.12 \mathrm{eV}$ need be considered. If all saddle points connecting those local minima were higher than $0.7 \mathrm{eV}$, then their populations would essentially be frozen over $t_{\text {avg }}$, and dissipation would again be negligible.

Dissipation occurs if some of the barriers lie between 0.4 and $0.7 \mathrm{eV}$. We then consider groups of local minima instead of individual minima. The minima within each group remain in local equilibrium if they are connected by barriers much less than $0.4 \mathrm{eV}$. The motion of the tip continuously changes the free energy of each group and drives certain groups into metastability if they are surrounded by high barriers. Dissipation occurs whenever such a group can suddenly relax into other lower free energy groups. Such an event becomes probable if under a certain tip-sample distance either a barrier connecting two groups continuously drops below $0.7 \mathrm{eV}$ or if a new lower barrier suddenly appears due to a connectivity change. Our simulations suggest that the latter mechanism is statistically more frequent. Saddle points and their connectivity are more sensitive to the tip-sample interaction than local minima.

We expect that groups of tip configurations which are in thermal equilibrium will also stabilize dynamically at finite temperature for distances of closest approach below or near $z_{c}$. In contrast to the $T=0$ case, the tip structure will switch between different configurations during $t_{\text {avg }}$. There is no well-defined tip structure, as was assumed in previous work. At $T=0, \Delta E$ was found to vary between 0 and $0.5 \mathrm{eV}$, depending on which tip structure is stabilized dynamically. At finite temperature, those variations will tend to average out over the sampled cycles. Taking a simple average over computed stabilized cycles as in Fig. 3, a rough estimate $\Delta E \approx 0.27 \mathrm{eV}$ is obtained.

In conclusion, nonequilibrium processes within silicon tips used in NCAFM are expected to dominate energy dissipation induced by short-range interactions with the sample. The softness of the frustrated apex structures facilitates hysteretic atomic rearrangements of tip atoms; this results in dissipation. Thermally activated jumps between temporarily equilibrated groups of tip configurations cause an intermittent redistribution of their populations and sudden energy losses which are, however, averaged over the time required to detect the dissipation. Stable, though somewhat noisy, atomically resolved images of topography and dissipation can then be simultaneously recorded in a limited range of temperature and distance $z$, as noticed in the case of diffusing surface species $[7,9]$. The phenomena discussed occur if short-range interactions are sufficiently strong to locally modify the potential energy landscape of the tip-sample system and will in general be samplespecific as well.

This work has been supported by the Swiss National Science Foundation and the Swiss National Center of Competence in Research (NCCR) on Nanoscale Science. Computer calculations were performed at the Swiss National Scientific Computing Center (CSCS) in Manno.

[1] S. Morita, R. Wiesendanger, and E. Meyer, Noncontact Atomic Force Microscopy, NanoScience and Technology (Springer-Verlag, Berlin, 2002).

[2] Y. Sugimoto et al., Phys. Rev. Lett. 98, 106104 (2007).

[3] See, for instance, Chap. 20 in Noncontact Atomic Force Microscopy, NanoScience and Technology (Ref. [1]).

[4] J.P. Cleveland, B. Anczykowski, A. E. Schmid, and V. B. Elings, Appl. Phys. Lett. 72, 2613 (1998).

[5] N. Sasaki and M. Tsukada, Jpn. J. Appl. Phys. 39, L1334 (2000).

[6] L. N. Kantorovich and T. Trevethan, Phys. Rev. Lett. 93, 236102 (2004).

[7] T. Trevethan et al., Nanotechnology 17, 5866 (2006), and references therein.

[8] T. Trevethan and L. Kantorovich, Nanotechnology 17, S205 (2006).

[9] M. Watkins, T. Trevethan, A. L. Shluger, and L. N. Kantorovich, Phys. Rev. B 76, 245421 (2007).

[10] Y. J. Li et al., Phys. Rev. Lett. 96, 106104 (2006).

[11] L. N. Kantorovich and C. Hobbs, Phys. Rev. B 73, 245420 (2006).

[12] N. Oyabu et al., Phys. Rev. Lett. 96, 106101 (2006).

[13] S. Goedecker, J. Chem. Phys. 120, 9911 (2004).

[14] T. J. Lenosky et al., Phys. Rev. B 55, 1528 (1997).

[15] W. Hellmann et al., Phys. Rev. B 75, 085411 (2007).

[16] L. Nony et al., Phys. Rev. B 74, 235439 (2006).

[17] G. H. Vineyard, J. Phys. Chem. Solids 3, 121 (1957).

[18] R. Hoffmann et al., Nanotechnology 18, 395503 (2007).

[19] F. Valiquette and N. Mousseau, Phys. Rev. B 68, 125209 (2003).

[20] A. Heyden, A. T. Bell, and F. J. Keil, J. Chem. Phys. 123, 224101 (2005).

[21] D. J. Wales, Energy Landscapes (Cambridge University Press, Cambridge, England, 2004).

[22] K.A. Dill and S. Bromberg, Molecular Driving Forces (Garland Science, Taylor and Francis, London, 2003). 